\begin{tabular}{|c|c|c|c|c|}
\hline & Variable & Nagelkerke & Drop & Rank \\
\hline \multirow[t]{5}{*}{ SITS } & ASPECTS & 0.080 & 0.039 & 1 \\
\hline & Number of Passes & 0.084 & 0.035 & 2 \\
\hline & IV tPA & 0.087 & 0.032 & 3 \\
\hline & Diabetes Mellitus & 0.093 & 0.025 & 4 \\
\hline & Lymphocytes & 0.098 & 0.021 & 5 \\
\hline \multirow[t]{7}{*}{ NINDS } & Neutrophils & 0.138 & 0.083 & 1 \\
\hline & Total WBC Count & 0.144 & 0.076 & 2 \\
\hline & IV tPA & 0.163 & 0.057 & 3 \\
\hline & Number of Passes & 0.171 & 0.049 & 4 \\
\hline & Diabetes Mellitus & 0.197 & 0.023 & 5 \\
\hline & Platelet to Lymphocyte & 0.201 & 0.020 & 6 \\
\hline & Ratio & & & \\
\hline Asymptomatic & Location of Clot & 0.170 & 0.065 & 1 \\
\hline \multicolumn{5}{|l|}{ Hemorrhage } \\
\hline & Onset to Perfusion & 0.175 & 0.060 & 2 \\
\hline & Total WBC Count & 0.182 & 0.053 & 3 \\
\hline & IV tPA & 0.202 & 0.033 & 4 \\
\hline & NIHSS Score & 0.209 & 0.025 & 5 \\
\hline & Prior Anticoagulants & 0.215 & 0.020 & 6 \\
\hline & Number of Passes & 0.223 & 0.012 & 7 \\
\hline \multirow[t]{6}{*}{ Overall Hemorrhage } & Total WBC Count & 0.156 & 0.059 & 1 \\
\hline & Number of Passes & 0.162 & 0.054 & 2 \\
\hline & Neutrophils & 0.176 & 0.039 & 3 \\
\hline & ASPECTS & 0.176 & 0.039 & 4 \\
\hline & Diabetes Mellitus & 0.178 & 0.038 & 5 \\
\hline & $\mathrm{CHF}$ & 0.200 & 0.016 & 6 \\
\hline
\end{tabular}

Disclosures J. Diestro: None. C. Parra-Farinas: None. M. Balas: None. Z. Zador: None. N. Almusalam: None. A. Dmytriw: None. P. Rajendram: None. R. Phillips: None. A. Alqabbani: None. E. Qazi: None. Y. Li: None. W. Montanera: None. D. Sarma: None. T. Marotta: None. A. Bharatha: None. J. Spears: None.

\section{P-004 NIHSS 24H AFTER MECHANICAL THROMBECTOMY PREDICTS 90-DAY FUNCTIONAL OUTCOME}

P Hendrix*, I Melamed, O Goren, R Zand, C Schirmer, C Griessenauer. Geisinger, Danville, $P A$

\subsection{6/neurintsurg-2021-SNIS.40}

Objective Mechanical thrombectomy (MT) for large vessel occlusion (LVO) ischemic stroke is a safe and effective treatment modality. The NIHSS 24 hours after MT (24h-NIHSS) was shown to serve as the strongest surrogate for 90-day functional outcome. The authors sought to externally validate 24h-NIHSS as predictor for 90-day functional outcome and explore additional variables in this context.

Methods Patients treated for anterior LVO between 02/2016 and $08 / 2020$ with premorbid mRS $<3$ were included. Receiver operating characteristics were used to compare different NIHSS-related surrogates such as baseline-NIHSS, 24hNIHSS, $\Delta$-NIHSS and percent-change-NIHSS to predict favorable function outcome (mRS 0-2). Additional analysis was performed to assess predictors associated with poor outcome despite reaching the best predictor threshold.
Results A total of 337 eligible cases were identified. 24hNIHSS outperformed B-NIHSS, $\Delta$-NIHSS, and $\%$-NIHSS in terms of 90 -day mRS $0-2$ prediction. $24-\mathrm{NIHSS} \leq 8$ was identified as the optimal binary threshold. Multivariable analysis demonstrated 24-NIHSS $\leq 8$, younger patient age, and shorter hospital stay independently associated with mRS $0-2$. Despite achieving 24h-NIHSS $\leq 8,23 / 143(16.1 \%)$ cases experienced poor outcome (mRS 4-6). Older age, higher baseline NIHSS, coexisting chronic kidney disease, and longer hospital stay were independent predictors for poor outcome despite achieving 24h-NIHSS $\leq 8$.

Conclusion NIHSS of 8 or less 24 hours after MT was validated to serve as an independent surrogate for favorable functional outcome. However, cofactors such as older age, higher baseline NIHSS and coexisting comorbidities appear to mitigate this clinical adjunct.

Disclosures P. Hendrix: None. I. Melamed: None. O. Goren: None. R. Zand: None. C. Schirmer: None. C. Griessenauer: None.

\section{P-005 CLOT SPATIAL HETEROGENEITY RATHER THAN HISTOLOGIC COMPOSITION IS RELATED TO FPE RATE}

Y Liu*, W Brinjikji, M Abbasi, D Dai, J Arturo Larco, D Kallmes, L Savastano. Mayo Clinic, Rochester, MN

\subsection{6/neurintsurg-2021-SNIS.41}

Introduction Characteristics of clots from stroke patients have gained a lot of interest in recent years as they could provide insights to improve the endovascular and medical management of ischemic stroke. Histology have been used to quantify the clot compositions, but analysis of clot structural pattern has been limited to qualitative description.

Methods From the STRIP (stroke thromboembolism registry of imaging and pathology) registry, we analyzed clots from 157 passes retrieved by mechanical thrombectomy from stroke cases where TICI $2 \mathrm{c} / 3$ was achieved. The clots were stained with Martius Scarlet Blue. To quantify the clot heterogeneity, we developed an algorithm to automatically segment scanned images into uniform grids and calculate the composition within each grid. Using the variance of compositions across neighboring grids, we proposed and implemented a formula to calculate the spatial heterogeneity index (SPI) value. To validate the clinical significance, we compared the clot SPI and clot compositions (red blood cells, fibrin, platelets, white blood cells) between cases with first pass effect (FPE) and cases without. SPI values of clots from cases with different etiologies, thrombectomy techniques, and tPA admission were also compared.

Results The average SPI value was 0.040 (SD 0.019) with a large range (0.001-0.091) with a grid size of $0.3 \mathrm{~mm}$. For cases with FPE $(\mathrm{n}=107)$, SPI was significantly smaller (0.038 vs $0.044, \mathrm{p}=0.05)$ compared to those without $(\mathrm{n}=$ 50). In comparison, none of the clot compositions was significantly different between cases with FPE and cases without. There was no significant difference in heterogeneity for clots from cases with different etiologies or retrieved with different thrombectomy techniques. Admission of tPA didn't change the clot heterogeneity either.

Conclusion SPI is a useful measure of clot structural heterogeneity and could provide more insights to improve the thrombectomy outcome compared to clot compositions. 